\title{
Streptococcus agalactiae: a vaginal pathogen?
}

\author{
A. N. MANIATIS, J. PALERMOS*, M. KANTZANOU*, N. A. MANIATIS*, C. CHRISTODOULOU* and \\ N. J. LEGAKIS $\dagger$
}

Department of Microbiology, Medical School, University of Athens and *Section of Infectious Diseases and Section of Clinical Microbiology, Hellenic Air Force and VA General Hospital, Athens, Greece

\begin{abstract}
The significance of Streptococcus agalactiae as an aetiological agent in vaginitis was evaluated. A total of 6226 samples from women who presented with vaginal symptoms was examined. The presence of $>\mathbf{1 0}$ leucocytes/high-power field (h.p.f.) was taken to be the criterion of active infection. $S$. agalactiae was isolated from $10.1 \%$ of these samples. The isolation rates of other common pathogens such as Candida spp., Gardnerella vaginalis and Trichomonas spp. were $54.1 \%, 27.2 \%$ and $4.2 \%$, respectively, in the same group of patients. In contrast, the isolation rates of these micro-organisms in the group of patients who had no infection $(<10$ leucocytes/h.p.f.) were $4.2 \%, 38.3 \%, 33 \%$ and $0.5 \%$, respectively. In the majority of samples from which $S$. agalactiae was isolated, it was the sole pathogen isolated $(83 \%)$ and its presence was associated with an inflammatory response in $80 \%$ of patients. Furthermore, the relative risk of vaginal infection with $S$. agalactiae $(2.38)$ in patients with purulent vaginal discharge was greater than that of Candida spp. infection (1.41) and lower than that of Trichomonas spp. infection (8.32). These data suggest that $S$. agalactiae in symptomatic women with microscopic evidence of inflammation should be considered a causative agent of vaginitis.
\end{abstract}

\section{Introduction}

Streptococcus agalactiae ( $\beta$-haemolytic streptococcus group B) is known to cause various infections in adults, but clinical interest in these bacteria mainly relates to their ability to cause serious neonatal illness, especially meningitis and sepsis. Although neonates born by caesarian section have presented with $S$. agalactiae infection, indicating ascending transmission of the micro-organism from the vagina of their mothers, in the majority of cases the neonate acquires the infection during labour through contact with the vaginal secretions of the mother colonised by $S$. agalactiae [1-6]. It is well known that $S$. agalactiae colonises the female genital tract but it is unclear if this bacterium can cause true infection of the vagina.

In this context, the frequency of $S$. agalactiae isolations in vaginal secretions of women with symptoms that included vaginal discharge, itching and a burning sensation was examined.

\section{Materials and methods}

A total of 6226 vaginal specimens taken from that

Received 9 Aug. 1995; accepted 16 Aug. 1995

†Corresponding author: N. J. Legakis. number of women who presented to the Gynaecology Department of the Hellenic Air Force Hospital complaining about vaginal discomfort, in the years 1992-1994 was examined. Their age range was 17-45 years. All specimens were obtained in a uniform manner by swabbing the posterior vagina, through a colposcope to enable a better view of the area. Swabs for microscopy were placed in $1 \mathrm{ml}$ of normal saline and swabs for culture in Stuart's transport medium. Processing of specimens was performed within $2 \mathrm{~h}$. Two specimens were collected from each patient, one for culture and the other for direct microscopy of a wet preparation followed by Gram's stain. Both stained and unstained preparations were examined for pus cells, epithelial cells, 'clue cells' and micro-organisms, especially Trichomonas spp. Culture media included blood agar, chocolate agar and Sabouraud agar. Each plate was streaked in such a manner as to allow estimation of the density of growth of the microorganisms [7]. Cultures were examined for growth of $S$. agalactiae, Candida spp. and Gardnerella vaginalis, as well as for growth in significant numbers of any other micro-organism. Streptococcal strains were identified by gram-staining, production of catalase, CAMP test, serogrouping and the API20 Strep system (API System, bioMérieux, France).

Three serotypes of $S$. agalactiae were identified (types I, II and III), by slide agglutination with latex particles 
sensitised with purified rabbit immunoglobulins specific for each of the three types (Pastorex B streptococci; Diagnostics Pasteur) [8].

The degree of association of various micro-organisms in causing vaginitis was assessed by the relative risk (RR) and population attributable risk (PAR) statistical parameters [9].

\section{Results}

A total of 6226 specimens from patients who presented with vaginal discomfort was submitted for culture; 4394 (65.6\%) were positive for potentially pathogenic micro-organisms. Candida spp. were the micro-organisms most frequently isolated $(34.2 \%)$, followed by $G$. vaginalis $(20.6 \%), S$. agalactiae $(5.7 \%)$ and Trichomonas spp. (3.2\%).

The presence of $>10$ leucocytes/high power field (h.p.f.) was taken as an objective criterion for

Table 1. Presence of micro-organisms in vaginal discharge and inflammatory response

\begin{tabular}{|c|c|c|c|c|}
\hline \multirow{2}{*}{$\frac{\text { Micro-organism }}{S . \text { agalactiae }}$} & \multicolumn{2}{|c|}{$\begin{array}{l}\text { Number }(\%) \\
\text { of cases with } \\
<10 \text { leucocytes }\end{array}$} & \multicolumn{2}{|c|}{$\begin{array}{c}\text { Number }(\%) \\
\text { of cases with } \\
>10 \text { leucocytes }\end{array}$} \\
\hline & 68 & (4.3) & 287 & $(10.1)$ \\
\hline Candida spp. & 598 & $(38.3)$ & 1534 & $(54.1)$ \\
\hline G. vaginalis & 517 & $(33.0)$ & 771 & $(27.2)$ \\
\hline Trichomonas spp. & 8 & $(0.5)$ & 118 & $(4.2)$ \\
\hline Enterobacteriaceae & 168 & $(10.8)$ & 62 & $(2.2)$ \\
\hline Enterococcus spp. & 115 & (7.4) & 41 & $(1.5)$ \\
\hline CNS & 56 & (3.6) & 17 & $(0.6)$ \\
\hline S. aureus & 30 & $(2.0)$ & 4 & $(0.1)$ \\
\hline Total & \multicolumn{2}{|c|}{$1560(100)$} & \multicolumn{2}{|c|}{$2834(100)$} \\
\hline
\end{tabular}

Table 2. Degree of association of various organisms with vaginal infection

\begin{tabular}{lcc}
\hline Micro-organism & $\begin{array}{c}\text { Relative } \\
\text { risk } \\
\text { (RR) }\end{array}$ & $\begin{array}{c}\text { Population } \\
\text { attributable } \\
\text { risk (PAR) }\end{array}$ \\
\hline S. agalactiae & 2.38 & 2.7 \\
Candida spp. & 1.41 & 7.18 \\
G. vaginalis & 0.82 & 2.6 \\
Trichomonas spp. & 8.32 & 1.6 \\
\hline
\end{tabular}

infection. Thus, according to this criterion, specimens were divided into two groups, those with $\geqslant 10$ leucocytes/h.p.f. and those with $<10$ leucocytes/h.p.f. group (Table 1). In the majority of cases, isolation of S. agalactiae, Candida spp. and Trichomonas spp. was associated with an inflammatory response. The presence of $S$. agalactiae was associated with a greater incidence of inflammatory response than that of Candida spp. and a lower incidence than that of Trichomonas spp. (Table 1). In contrast, the isolation from 4394 samples of 493 strains of micro-organisms considered as commensals, such as Enterobacteriaceae, staphylococci and enterococci, and included in the study only for statistical purposes, did not correlate with the presence of inflammatory cells (Table 1). In regard to the association of various organisms with vaginitis, the RR was $2.38,1.41$ and 8.32 for $S$. agalactiae, Candida spp., and Trichomonas spp., respectively, and the PAR was 2.7, 7.18 and 1.6, respectively (Table 2 ).

In $295(83 \%)$ of 355 cases, S. agalactiae was isolated as the sole pathogen. There were 60 cases $(17 \%)$ of mixed infections, in which $S$. agalactiae was isolated along with Candida spp., G. vaginalis or Trichomonas spp. (Table 3).

The serotype distribution of $S$. agalactiae isolates is shown in Table 4. Serotypes I and III were more commonly associated with an inflammatory response.

\section{Discussion}

Until now, $S$. agalactiae has been regarded as a normal component of the vaginal flora, associated with premature birth, amnionitis, neonatal meningitis and sepsis, but recent studies have concluded that invasive infections caused by $S$. agalactiae are not uncommon and that they pose a major problem not only in pregnant women and neonates but also in non-pregnant adults, especially the elderly and patients with chronic diseases [1-6].

According to current evidence, the gastrointestinal tract is the primary colonisation site. This is supported

Table 3. Mixed vaginal infections associated with $S$. agalactiae

\begin{tabular}{|c|c|c|c|c|}
\hline \multirow[b]{3}{*}{ Micro-organisms } & \multicolumn{4}{|c|}{ Number of specimens } \\
\hline & \multicolumn{3}{|c|}{ with leucocytes (/h.p.f.) } & \multirow[b]{2}{*}{ Total } \\
\hline & $10-20$ & $20-50$ & $>50$ & \\
\hline S. agalactiae & 136 & 92 & 6 & 234 \\
\hline S. agalactiae + Candida spp. & 15 & 9 & 4 & 28 \\
\hline S. agalactiae $+G$. vaginalis & 5 & 5 & - & 10 \\
\hline S. agalactiae + Trichomonas spp. & 2 & 8 & 1 & 11 \\
\hline S. agalactiae + Trichomonas spp. + Candida spp. & - & 1 & 1 & 2 \\
\hline S. agalactiae + Trichomonas spp. + G. vaginalis & - & 1 & - & 1 \\
\hline Total & 158 & 116 & 12 & 286 \\
\hline
\end{tabular}


Table 4. Distribution of $S$. agalactiae serotypes (\%)

\begin{tabular}{lcc}
\hline & \multicolumn{2}{c}{ Number with leucocytes (/h.p.f.) } \\
\cline { 2 - 3 } Serotype & $<10(\mathrm{n}=68)$ & $>10(\mathrm{n}=287)$ \\
\hline I & 37 & 36 \\
II & 37 & 16 \\
III & 17 & 34 \\
NT & 5 & 15 \\
\hline
\end{tabular}

NT, not typable.

by the relatively high isolation rates of $S$. agalactiae from cultures of the anal region, compared to the genital region [3]. An estimated $40 \%$ of pregnant women carry the organism throughout their pregnancy, but transient vaginal colonisation may be more frequent [3]. In a survey in Israel on pre-term Israeli and Arabic women, the colonisation rates were 5.4\% and $1.6 \%$ respectively [10]. A similar study in Germany revealed a $3.8 \%$ rate [11]. In three Italian hospitals, $7.5 \%$ of mothers and $4.9 \%$ of babies were found to be colonised [12]. Higher rates of colonisation were reported in England (28\%) [13] and in the USA $(20 \%)[14]$.

The role of $S$. agalactiae as an aetiological agent of vaginitis has not yet been established. Studies dealing with the organism as an infectious agent in bacterial vaginitis are limited either in the manner of reporting numbers of positive isolates or in epidemiological considerations concerning colonisation of the genital or gastrointestinal tract $[15,16]$. Vaginitis is a common gynaecological disease, characterised by objective and subjective signs of infection. The presence of leucocytes, combined with clinical symptoms, may be considered an objective criterion of infection. The exception to this is bacterial vaginosis where the absence of an inflammatory response may be due to the production of succinate, an inhibitor of chemotaxis, by Bacteroides urealyticus and Prevotella bivia [17].

This study evaluated the possible pathogenic role of $S$. agalactiae based on the criterion of the presence of $>10$ leucocytes/h.p.f. in the vaginal secretions to denote infection of the vagina and not only on the presence of clinical symptoms. $S$. agalactiae was isolated from $10.1 \%$ of the specimens with $>10$ leucocytes/h.p.f. and from $4.3 \%$ of the specimens with $<10$ leucocytes/h.p.f. We strongly believe that $S$. agalactiae is as pathogenic as the other established pathogens and should be considered a vaginal pathogen for the following reasons. Firstly, the recovery rate of $S$. agalactiae in the leucocyte-positive group was much higher than in the leucocyte-negative group. The association between $S$. agalactiae and symptomatology was at least as high as that of Candida spp. and slightly lower than that of Trichomonas spp. Secondly, in the majority of specimens from which $S$. agalactiae was isolated $(83 \%)$ it was the sole pathogen. There- fore, it is reasonable to consider it as the cause of the infection.

Differences in the prevalence of $S$. agalactiae internationally may be attributed to different methods of specimen collection, transport media and isolation methods. In the present study chocolate and blood agar were used exclusively as culture media. Use of selective solid culture media such as the Columbia colistin-nalidixic acid (CNA) agar and enrichment broth (Todd-Hewitt supplemented with gentamicin $8 \mu \mathrm{g} / \mathrm{ml}$ ) may have yielded higher isolation rates [18].

Irrespective of the patient's age, the distribution of isolates was not divided evenly among the three major serotypes (I, II, and III), as has been reported for asymptomatically colonised patients [6]. Serotypes I and III were present more than twice as often as serotype II in the leucocyte-positive group.

Although no resistance to penicillin was observed, treatment failures have been reported when penicillin was used to eradicate group B streptococci from the genital tract of women [19]. This has brought about the use of erythromycin in empirical antimicrobial treatment. In a previous study, $9 \%$ of strains were found to be resistant to erythromycin [20]. Such strains have also been detected in other studies but with a lower frequency [21-24]. This resistance to erythromycin may be related its extensive use in the empirical treatment of female lower genital tract infections [20]. Susceptibility testing for $S$. agalactiae is not usually performed as the species remains susceptible to penicillins. However, high rates of penicillin treatment failures as well as the resistance rates to erythromycin suggest that the antibiotic susceptibility trends of these pathogens should be monitored carefully.

\section{References}

1. Ross PW. Group-B streptococcus - profile of an organism. J Med Microbiol 1984; 18: 139-166.

2. Regan JA, Cha S, James LS. Premature rupture of membranes, preterm delivery, and group B streptococcal colonization of mothers. Am J Obstet Gynecol 1981; 141: 184-186.

3. Boyer KM, Gadzala CA, Kelly PD, Burd LI, Gotoff SP. Selective intrapartum chemoprophylaxis of neonatal group B streptococcal early-onset disease. II. Predictive value of prenatal cultures. $J$ Infect Dis 1983; 148: 802-809.

4. Schwartz B, Schuchat A, Oxtoby MJ, Cochi SL, Hightower A, Broome CV. Invasive group B streptococcal diseases in adults. A population-based study in metropolitan Atlanta. JAMA 1991; 266: $1112-1114$.

5. Farley MM, Harvey RC, Stull $\mathrm{T}$ et al. A population-based assessment of invasive disease due to group B streptococcus in nonpregnant adults. $N$ Engl $J$ Med 1993; 328: 1807-1811.

6. Edwards MS, Baker CJ. Streptococcus agalactiae (group B streptococcus). In: Mandell GL, Douglas RG, Bennet JE (eds) Principles and practice in infectious diseases, 3rd edn. New York, Churchill Livingstone, 1990. 1554-1563.

7. Wald ER, Dashefsky B, Green $M$ et al. Rapid detection of group B streptococci directly from vaginal swabs. $J$ Clin Microbiol 1987; 25: 573-574. 
8. Colman G. Typing of Streptococcus agalactiae (Lancefield Group B). Eur J Clin Microbiol Infect Dis 1988; 7: 226-231.

9. Fletcher RH, Fletcher SW, Wagner EH (eds). Clinical epidemiology - the essentials. Baltimore, Williams and Wilkins. 1982: 91-105.

10. Eidelman AI, Rudensky B, Turgeman D, Nubani N, Schimmel MS, Isacsohn M. Epidemiology of group B streptococci colonization and disease in mothers and infants: update of ongoing 10-year Jerusalem study. Isr J Med Sci 1990; 26: 7173.

11. Forster U, Borkhardt HL. [Vaginal streptococcus B colonization in pregnancy.] Zentralbl Gynakol 1988; 110: 174-178.

12. Visconti A, Orefici G, Notarnicola AM. Colonization and infection of mothers and neonates with group B streptococci in three Italian hospitals. J Hosp Infect 1985; 6: 265-276.

13. Easmon CS, Hastings JGM, Neill J, Bloxham B, Rivers RPA. Is group B streptococcal screening during pregnancy justified? Br J Obstet Gynaecol 1985; 92: 197-201.

14. Anthony BF, Eisenstadt R, Carter J, Kim KS, Hobel CJ. Genital and intestinal carriage of Group B streptococci during pregnancy. J Infect Dis 1981; 143: 761-766.

15. Jensen NE, Andersen BL. The prevalence of group B streptococci in human urogenital secretions. Scand J Infect Dis 1979; 11: 199-202.

16. Savarino A, Mattei R, Barsotti A, Bechi R, Saisi F, Lenci F. [Hemolytic group $B$ streptococcus in cervico-vaginal secretions.] Quad Sclavo Diagn 1988; 24: 185-188.

17. Catlin BW. Gardnerella vaginalis: characteristics, clinical considerations, and controversies. Clin Microbiol Rev 1992; 5: 213-237.

18. Kontnick CM, Edberg SC. Direct detection of group B streptococci from vaginal specimens compared with quantitative culture. J Clin Microbiol 1990; 28: 336-339.

19. Gardner SE, Yow MD, Leeds LJ, Thompson PK, Mason EO, Clark DJ. Failure of penicillin to eradicate group B streptococcal colonization in the pregnant woman. A couple study. Am J Obstet Gynecol 1979; 135: 1062-1065.

20. Tsakris A, Maniatis AN. Inducible type of erythromycin resistance among group B streptococci isolated in Greece. J Antimicrob Chemother 1993; 31: 177-178.

21. Kim KS. Antimicrobial susceptibility of GBS. Antibiot Chemother 1985; 35: 83-89.

22. Aronoff SC, Laurent C, Jacobs MR. In-vitro activity of erythromycin, roxithromycin and CP 62993 against common paediatric pathogens. J Antimicrob Chemother 1987; 19: 275276.

23. Von Recklinghausen G, Fischer W, Schmidt K, Ansorg R. Resistance of Streptococcus agalactiae to erythromycin. $J$ Antimicrob Chemother 1989; 24: 93.

24. Berkowitz K, Regan JA, Greenberg E. Antibiotic resistance patterns of group B streptococci in pregnant women. J Clin Microbiol 1990; 28: 5-7. 